\title{
Efectividad del ejercicio físico como intervención coadyuvante en las adicciones: una revisión
}

\section{Effectiveness of exercise as a complementary intervention in addictions: a review}

\author{
Núria Siñol*; Ester Martínez-SÁnChez ${ }^{\star *}$; Elisabeth \\ GuILlamó ${ }^{* * *}$; M. Josefa Campins*; Fàtima Larger*; \\ JOAN TRUJOLS ${ }^{\star},{ }^{\star \star \star \star *}$
}

\author{
Unitat de Conductes Addictives, Servei de Psiquiatria, Hospital de la Santa \\ Creu i Sant Pau, Institut d'Investigació Biomèdica Sant Pau (IIB Sant Pau), \\ Barcelona \\ ** CAP Florida Sud, L'Hospitalet de Llobregat, Barcelona \\ *** Facultat de Medicina, Departament de Ciències Fisiològiques II, Unitat \\ Fisiologia i Fisiopatologia de l'Exercici, Universitat de Barcelona (UB Bellvitge), \\ L'Hospitalet de Llobregat, Barcelona \\ ${ }_{\star \star \star * \star}$ Centro de Investigación Biomédica en Red de Salud Mental (CIBERSAM), \\ Madrid \\ Enviar correspondencia a: \\ Núria Siñol \\ Unitat de Conductes Addictives, Servei de Psiquiatria, \\ Hospital de la Santa Creu i Sant Pau, \\ Institut d'Investigació Biomèdica Sant Pau (IIB Sant Pau) \\ C/ Sant Antoni Maria Claret, 167 \\ 08025 Barcelona \\ nsinol@santpau.cat
}

recibido: Julio 2012 aceptado: Diciembre 2012

\section{Resumen}

Dados los beneficios que aporta el ejercicio físico sobre la salud física y mental, varios autores han explorado la efectividad del mismo como tratamiento en diversas patologías, entre ellas los trastornos adictivos. El objetivo de este artículo fue revisar la literatura existente sobre la efectividad del ejercicio físico como intervención coadyuvante en el tratamiento de la dependencia de sustancias psicoactivas. Para conseguir dicho objetivo se realizó una búsqueda bibliográfica en las siguientes bases de datos: PubMed, Scopus, ISI Web of Knowledge, The Cochrane Library, TESEO y TDX. Se seleccionaron aquellos artículos que evaluaban la efectividad del ejercicio físico sobre el craving, la sintomatología de abstinencia, el mantenimiento de la abstinencia y en el caso de la dependencia de nicotina también aquellos que evaluaban el efecto del ejercicio sobre el aumento de peso al dejar de fumar. Diecisiete artículos cumplieron los criterios de selección. La utilidad del ejercicio físico como estrategia coadyuvante en los tratamientos habituales para la dependencia de sustancias psicoactivas parece demostrada, tanto para favorecer la reducción del consumo como para facilitar la disminución del craving y la sintomatología de abstinencia, especialmente en el caso de la dependencia de nicotina. Sin embargo, especialmente en el caso de la dependencia de sustancias psicoactivas ilegales, son necesarios más estudios para llegar a resultados concluyentes. Destacar igualmente que se trata de un tipo de intervención bien aceptada por parte de la población adicta, hecho que la convertiria en una herramienta especialmente útil en personas en tratamiento de su trastorno adictivo.

Palabras clave: Ejercicio físico, actividad física, intervención, dependencia de sustancias, tratamiento

\section{Abstract}

Given the benefits that exercise brings to physical and mental health, several authors have investigated its effectiveness as a treatment for various pathologies such as addictive disorders. The aim of this paper is to review the literature regarding the effectiveness of exercise as a complementary intervention for treatment of psychoactive substance dependence. To achieve this aim, a bibliographic search was conducted in the following databases: PubMed, Scopus, ISI Web of Knowledge, The Cochrane Library, TESEO and TDX. Those articles which evaluated the effectiveness of exercise on craving, withdrawal symptoms and abstinence rates were selected. Regarding nicotine dependence, those studies which evaluated the effectiveness of exercise for reducing weight gain after quitting smoking were also chosen. Seventeen articles met the selection criteria. The usefulness of exercise as a complementary strategy along with habitual therapeutic treatments for psychoactive substance dependence seems to be demonstrated, especially in nicotine dependence for the following variables: achieving or maintaining abstinence, and reducing craving or withdrawal symptoms. Further studies are nevertheless required, especially on illegal psychoactive substance dependence to be able to achieve conclusive results. We must also equally note that this kind of intervention is widely accepted by the drug using community, fact that would make of it a particularly useful tool in treating people with addictive disorders.

Key Words: Exercise, physical activity, intervention, substance dependence, treatment 
$\mathrm{D}$ ados los numerosos beneficios que el ejercicio físico aporta tanto sobre la salud física (p.ej. United States Department of Health and Human Services [USDHHS], 1996) como sobre la salud mental (Fox, 1999; Penedo y Dahn, 2005; Taylor y Faulkner, 2008; Taylor, Sallis y Needle, 1985), varias instituciones han confeccionado guias con prescripciones de ejercicio físico para la promoción de la salud y la prevención de enfermedades en la población general (Bull y the Expert Working Groups, 2010; Generalitat de Catalunya, 2007; USDHHS, 2008a; Whitelaw, Swift, Goodwin y Clark, 2008). Paralelamente, se han delimitado las directrices y recomendaciones sobre el tipo, cantidad e intensidad de ejercicio adecuado para que se consigan estos efectos beneficiosos sobre la salud (Blair, LaMonte y Nichaman, 2004; Haskell et al., 2007; Pate et al., 1995; USDHHS, 2008b).

Asimismo, a lo largo de los últimos años, un número importante de autores han explorado la eficacia del ejercicio físico en el tratamiento de diversas patologías, tanto médicas (Pedersen y Saltin, 2006) como psiquiátricas (Daley, 2002; Stathopoulou, Powers, Berry, Smits y Otto, 2006; Wolff et al., 2011), elaborándose guias y manuales específicos (Durstine, Moore, Painter, y Roberts, 2009; Generalitat de Catalunya, 2009; Moore, 2004). Concretamente, en el ámbito de las enfermedades psiquiátricas, se ha comprobado la utilidad del ejercicio físico como tratamiento complementario en el abordaje de la depresión (Dimeo, Bauer, Varahram, Proest y Halter, 2001; Knubben et al., 2007; Lawlor y Hopker, 2001; Mead et al., 2009), la esquizofrenia (Holley, Crone, Tyson y Lovell, 2011) y el trastorno bipolar (Wright, Everson-Hock y Taylor, 2009), entre otros.

En relación con el consumo de sustancias psicoactivas, no solo se ha observado una asociación negativa entre la práctica regular de ejercicio físico y el consumo de sustancias (Goodwin, 2003; Kaczynski, Manske, Mannell y Grewal, 2008; Pate, Heath, Dowda y Trost, 1996), sino que además se ha identificado la ausencia de actividad física como una variable predictora del consumo de sustancias psicoactivas en la adolescencia (Aveyard y Ussher, 2007; Korhonen, Kujala, Rose y Kaprio, 2009; Kujala, Kaprio y Rose, 2007). Asimismo, el ejercicio físico regular ha sido sugerido como una actividad alternativa al consumo de sustancias psicoactivas, siendo altamente recomendado como elemento facilitador del cambio de estilo de vida (Marlatt, 1985), así como estrategia útil para la prevención de recaídas (deRuiter y Faulkner, 2006).

Entre los mecanismos mediadores y/o explicativos de los efectos positivos del ejercicio en el tratamiento de la dependencia de sustancias psicoactivas (Marlatt, 1985; Read y Brown, 2003), se encuentran: (i) conseguir estados placenteros similares a los producidos por el consumo, mediante la activación de los mecanismos de neurotransmisión y las áreas de refuerzo, (ii) la reducción de la sintomatología ansiosa y depresiva inducida durante la abstinencia y, en general, la mejora de los estados de ánimo, (iii) el incremento de la autoeficacia, (iv) la promoción del ejercicio como una alternativa positiva al consumo, con el objetivo de progresar hacia un cambio de estilo de vida más saludable, (v) el empleo del ejercicio como estrategia de afrontamiento y reducción del estrés, (vi) como técnica que facilite la reducción del craving y, (vii) como estrategia para incrementar las relaciones interpersonales y el apoyo social cuando el ejercicio se realiza en grupo.

Debido a la ausencia de una revisión que sintetice la evidencia disponible sobre la utilidad del ejercicio físico como intervención coadyuvante en el tratamiento de la dependencia de las diferentes sustancias psicoactivas tanto legales como ilegales, el presente artículo tiene como objetivo revisar la literatura existente sobre la efectividad del ejercicio físico como intervención coadyuvante en el tratamiento de la dependencia de sustancias psicoactivas.

\section{Método}

Para la elaboración de esta revisión se realizó una búsqueda bibliográfica en profundidad en las siguientes bases de datos: MEDLINE/PubMed, Scopus, ISI Web of Knowledge y The Cochrane Library. Asimismo, se realizó una búsqueda de la denominada literatura gris mediante el motor de búsqueda Buscador Web de Google y en las bases de datos de tesis doctorales TESEO y TDX. Con el objetivo de identificar estudios relevantes adicionales, se revisaron además las referencias de los artículos seleccionados.

Para la búsqueda electrónica en dichas bases de datos bibliográficas se utilizó una combinación de tres subgrupos diferentes de términos. El primer subgrupo estaba formado por los términos "physical activity" o "exercise". El segundo subgrupo lo formaban los términos "treatment" y "substance abuse" o "substance dependence". Finalmente, el tercer grupo estaba formado por términos específicos por sustancias: "smok", "alcohol", "cocaine", "cannabis", "opioid" o "heroin". El primer subgrupo se combinó con los otros dos.

A partir del resumen se seleccionaron aquellos artículos que evaluaban la efectividad del ejercicio físico como intervención coadyuvante en el tratamiento de la dependencia de sustancias psicoactivas y evaluaban el efecto del ejercicio sobre: (i) el craving, (ii) la sintomatología de abstinencia, (iii) el mantenimiento de la abstinencia en el tratamiento de la dependencia de sustancias psicoactivas y, (iv) en el caso de la dependencia de nicotina, el aumento de peso al dejar de fumar.

Aunque no se aplicaron restricciones de fecha de publicación y la búsqueda bibliográfica finalizó en el mes de enero de 2012, sí hubo restricciones relacionadas con el idioma, limitándose la búsqueda a trabajos publicados en castellano, inglés y francés. Además, la población objeto de estudio debia ser adulta, excluyéndose aquellos estudios con población infantil, adolescente 0 anciana. Asimismo, se excluyeron los trabajos donde la intervención de ejercicio físico se realizaba en una sola sesión o cuando no formaba parte de un tratamiento de intervención para la dependencia de sustancias psicoactivas. De los 239 artículos inicialmente identificados, 17 cumplieron los criterios de inclusión. 


\section{Resultados}

\section{El ejercicio físico en el tratamiento de la dependencia de nicotina}

La mayoría de los intentos para dejar de fumar se realizan sin ayuda profesional, pero solo entre un 3-5\% de las personas que lo intentan logran mantener la abstinencia más allá de los 6 meses, produciéndose la mayoría de las recaídas durante los primeros días después de cesar el consumo (Hughes, Keely y Naud, 2004). Con el objetivo de mejorar estos índices, en las últimas décadas se han desarrollado intervenciones para el tratamiento del tabaquismo y prevenir las recaídas (Hajek, Stead, West, Jarvis y Lancaster, 2009), aunque la eficacia de las mismas es limitada y dichas intervenciones siguen presentando elevados índices de recaída (Hajek et al., 2009; Piasecki, 2006).

Entre las variables asociadas de forma consistente con la recaída del consumo de tabaco destacan la sintomatología de abstinencia y el deseo de consumo o craving (Hughes, 2006; Piasecki, 2006). Asimismo, sobretodo en mujeres, la preocupación y el miedo a aumentar de peso al dejar de fumar ha mostrado ser tanto un elemento motivador para no abandonar el consumo de tabaco (USDHHS, 2001) como un factor de riesgo para la recaída en dicho consumo (Clark et al., 2006; Pisinger y Jorgensen, 2007).

A continuación, se revisan los estudios que evalúan la efectividad del ejercicio físico en (i) la reducción de la sintomatología de abstinencia y el craving de tabaco, (ii) en la reducción del aumento de peso al dejar de fumar y (iii) en el mantenimiento de la abstinencia de nicotina. En la tabla 1 se exponen cronológicamente los ensayos clínicos aleatorizados que utilizan un programa de ejercicio físico como intervención coadyuvante en el tratamiento de la dependencia de nicotina. Una revisión anterior centrada en el craving y la sintomatología de abstinencia puede hallarse en Taylor y Ussher (2005). Asimismo, una revisión sistemática actualizada, focalizada en la consecución y mantenimiento de la abstinencia puede consultarse en Ussher, Taylor y Faulkner (2012).

\section{Efecto del ejercicio físico en la reducción de la sintomatología de abstinencia y e/ craving de tabaco.}

La sintomatología de abstinencia de nicotina, tal y como se describe en el texto revisado del Manual diagnóstico y estadístico de los trastornos mentales (DSM-IV-TR) incluye cuatro o más de las siguientes variables (American Psychiatric Association [APA], 2002): estado de ánimo disfórico o depresivo, insomnio, irritabilidad, frustración o ira, ansiedad, dificultades de concentración, inquietud, disminución de la frecuencia cardíaca y aumento del apetito o del peso. Aunque el craving no se considera una variable propia de la sintomatología abstinencial, el mismo manual le concede un elevado grado de importancia diciendo que: "La necesidad irresistible (craving) es un elemento importante en la abstinencia de nicotina y puede explicar las dificultades de los sujetos para dejar de consumir productos que la contienen." (APA, 2002, p. 307). En cualquier caso, el craving es un elemento central en el tratamiento de las adicciones (Iraurgi y Corcuera, 2008).

El ejercicio físico se ha postulado como estrategia para reducir tanto la sintomatología de abstinencia como el craving de tabaco, debido a los efectos que ha demostrado tener sobre estados psicológicos como el malestar en general, la ansiedad y el estrés, entre otras (Faulkner y Taylor, 2005, 2009; Márquez, 1995). En este sentido, Bock, Marcus, King, Borrelli y Roberts (1999) examinaron el efecto agudo (cambios ocurridos justo después de la sesión de tratamiento) y crónico (cambios ocurridos entre el inicio y final de tratamiento) de un programa de ejercicio físico sobre la sintomatología de abstinencia y el craving de tabaco en mujeres sedentarias incluidas en un programa de cesación tabáquica. Dichas mujeres fueron asignadas aleatoriamente al grupo que realizaba ejercicio físico o al grupo control. El grupo de ejercicio físico realizaba, además de terapia cognitiva-conductual para dejar de fumar, un programa supervisado de 12 semanas de duración, consistente en 3 sesiones semanales de 45-60 minutos de duración y de intensidad vigorosa. El grupo control recibia la terapia cognitiva-conductual para dejar de fumar y asistía a sesiones informativas sobre salud y bienestar. Los resultados mostraron una reducción significativamente superior de la sintomatología de abstinencia y el craving de tabaco en el grupo de ejercicio, aunque solo en el caso del efecto agudo en cada sesión de tratamiento y no en el del efecto crónico.

En otro trabajo más reciente, Williams et al., (2011) observaron una tendencia hacia la significación estadistica en la reducción del craving pre-post sesión en el grupo de ejercicio. Aunque dicha reducción no se mantenía de sesión a sesión, cabe señalar que el programa de ejercicio, en comparación con el realizado por Bock et al., (1999), era de menor duración e intensidad (tres sesiones semanales de 50 minutos de ejercicio aeróbico de intensidad moderada durante 8 semanas). Incluso sin la supervisión in situ de la ejecución del programa de ejercicio, Ussher, West, McEwen, Taylor y Steptoe (2003) observaron una reducción de la sintomatología de abstinencia durante la primera semana después de dejar de fumar aplicando counseling para aumentar el nivel de actividad física y Bize et al., (2010) observaron una tendencia a la significación en la reducción de la depresión al final de una intervención de 10 semanas de duración en la que programaban el ejercicio para realizarlo la mayor parte del tiempo en casa.

Si bien el objetivo de la revisión se centra en el efecto del ejercicio físico en el tratamiento de la dependencia de sustancias para el mantenimiento de la abstinencia y no en la abstinencia temporal, una línea de investigación con notable producción científica es la que evalúa el impacto de una sola sesión de ejercicio en la reducción de la sintomatología de abstinencia y el craving de tabaco en fumadores sedentarios abstinentes de forma temporal (durante unas 15 horas). Puede consultarse la revisión de Taylor, Ussher y Faulkner (2007) para una presentación pormenorizada de los resultados de dicha línea de investigación. No obstante, a continuación resumimos sucintamente los principales resultados de dichos trabajos debido a su relevancia de cara a estudios posteriores.

En un primer estudio, Ussher, Nunziata, Cropley y West (2001) evaluaron el efecto que tenían 10 minutos de ejercicio 
aeróbico de intensidad moderada (mediante bicicleta estática) en la intensidad del deseo de fumar, así como en variables del síndrome de abstinencia en comparación con un grupo control que no recibía dicha intervención. Los resultados mostraron reducciones significativamente superiores, tanto de la intensidad del deseo de fumar como de la mayoría de variables de sintomatología de abstinencia evaluadas (excepto en la variable 'hambre'), en el grupo que realizaba ejercicio, hasta 20 minutos después del ejercicio.

El mismo grupo analizó si una menor duración (5 minutos de ejercicio en lugar de 10) a diferentes intensidades (baja 0 moderada) de ejercicio era suficiente para producir efectos positivos sobre dichas variables, mediante la asignación aleatoria de los participantes a una de las tres condiciones: control, ejercicio a intensidad baja y ejercicio a intensidad moderada (Daniel, Cropley, Ussher y West, 2004). En este caso, se confirmó que solo 5 minutos de ejercicio aeróbico tenían un efecto inmediato en la reducción del craving durante y después del ejercicio, aunque solo cuando la intensidad de la actividad era moderada y no a intensidades bajas. Algunas de las variables de abstinencia disminuyeron de forma significativa, aunque la efectividad de solo 5 minutos fue inferior a la encontrada en el estudio previo (Ussher et al., 2001) con el doble de duración. Asimismo, no se hallaron diferencias en la reducción del craving y sintomas de abstinencia entre el grupo de intensidad baja y el grupo control (Daniel et al., 2004). No obstante, Taylor, Katomeri y Ussher (2005) sí consiguieron mostrar el efecto positivo del ejercicio aeróbico de baja intensidad en el deseo de fumar. En este estudio los participantes debían recorrer una milla $(1,6 \mathrm{~km})$ caminando a su intensidad preferida. El tiempo medio empleado fueron unos 20 minutos a una intensidad de ejercicio baja (12,5 minutos el $\mathrm{km})$.

Dado que no todas las situaciones son aptas para realizar ejercicio aeróbico, el grupo de Ussher evaluó si el trabajo de fuerza mediante ejercicios isométricos era igual de efectivo que el ejercicio cardiovascular en la reducción de la sintomatología abstinencial y el craving de tabaco, mediante dos estudios donde se realizaban sesiones de 5 (Ussher, West, Doshi y Sampuran, 2006) y 10 (Ussher, Cropley, Playle, Mohidin y West, 2009) minutos de ejercicio. Los resultados mostraron que una sola sesión de 5 minutos de ejercicios isométricos era suficiente para disminuir el deseo de fumar hasta 5 minutos después del ejercicio en comparación con el grupo control; y como 10 minutos del mismo tipo de ejercicio, reducía además variables de sintomatología de abstinencia hasta 30 minutos después de la intervención. Sin embargo, estos resultados no fueron tan clínicamente significativos como los obtenidos anteriormente con ejercicio aeróbico.

\section{Efecto del ejercicio físico en la reducción del aumento de peso al dejar de fumar.}

La ganancia de peso tras el abandono del consumo de tabaco ha sido consistentemente mostrado en numerosos estudios (p.ej., Caan et al., 1996; Klesges et al., 1997; Travier et al., 2012; USDHHS, 1990). Aunque el aumento de peso varía en función de variables como la edad, las características del consumo de tabaco, el peso antes de dejar de fumar, el peso ganado en intentos anteriores, el tipo de dieta y los hábitos saludables previos (Pinto et al., 1999; Pomerleau, Zucker y Stewart, 2001), la preocupación por el peso es común entre los fumadores, situándose la prevalencia de fumadores preocupados por ganar peso al dejar de fumar en un $50 \%$ en el caso de las mujeres y en un $25 \%$ en el de los hombres (Clark et al., 2006).

La creencia de que el tabaco ejerce un control sobre el peso y sobre las emociones negativas, así como el miedo a ganar peso al dejar de fumar son variables que se asocian, especialmente en las mujeres, al inicio del consumo de tabaco, a su mantenimiento y a la recaída en él después de abandonar dicho consumo (Pisinger y Jorgensen, 2007; Pomerleau et al., 2001; USDHHS, 2001). Sin embargo, los mecanismos por los cuales existe esta ganancia de peso tras la abstinencia no están del todo claros, aunque entre ellos se incluyen el aumento del consumo de calorías y la disminución de la tasa metabólica en reposo (Caan et al., 1996; Filozof, Fernandez Pinilla y Fernandez-Cruz, 2004).

Un estudio realizado únicamente con enfermeras (Kawachi, Troisi, Rotnitzky, Coakley y Colditz, 1996) evaluó, en aquellas que habian dejado de fumar, si habían modificado su nivel de actividad física y si este influia en la ganancia ponderal. Aquellas que incrementaron su nivel de actividad física aumentaron menos de peso en comparación con aquellas que no habian modificado su nivel de actividad física. Al ajustar los datos por variables potencialmente confusoras, los resultados indicaron que aquellas mujeres que no modificaron su nivel de actividad física aumentaron una media de 2,3 y 4,5 kg en función de si fumaban menos de 25025 o más cigarrillos al dia, respectivamente. En el caso de las mujeres que incrementaron su nivel de actividad física en 8-16 METs (Unidad Metabólica de Reposo) a la semana (equivalente a 1-2 horas de actividad vigorosa), la media del aumento de peso fue de $1,8 \mathrm{~kg}$ en las que fumaban menos de 25 cigarrillos al dia y de $3,9 \mathrm{~kg}$ en las que fumaban 25 o más cigarrillos al día. Aquellas que aumentaron su nivel de actividad física en más de 16 METs a la semana presentaron una ganancia de peso de 1,3 $\mathrm{kg}$ o de 2,9 kg en función de si fumaban menos de 25 cigarrillos al día 025 o más cigarrillos al dia, respectivamente.

En un estudio realizado con posterioridad, Marcus et al., (1999) evaluaron el efecto que tenía un programa de ejercicio físico en el aumento de peso al dejar de fumar. Aunque las participantes que se mantuvieron sin fumar aumentaron significativamente de peso (durante el tratamiento y en el seguimiento a 3 y 12 meses) en comparación con aquellas que no lograron mantener la abstinencia, dicho aumento fue inferior $(3,1 \mathrm{~kg}$ vs. $5,4 \mathrm{~kg})$ en el grupo que realizaba ejercicio físico en comparación con el grupo control. En cambio, no se hallaron diferencias significativas entre grupos en aquellas participantes que no habian logrado dejar de fumar. El efecto del tratamiento, sin embargo, no se mantuvo en el seguimiento a 3 y 12 meses, cuando ya no se realizaba el programa de ejercicio de forma supervisada. En esta misma línea, estudios posteriores que han intentado aumentar el nivel de actividad física a través de counseling (Ussher, West, McEwen, Taylor y Steptoe, 2007) o mediante intervención motivacional (Bize et al., 2010) han mostrado una tendencia a la significación a favor del menor aumento de peso en los participantes asignados al grupo de ejercicio. 
Considerando la contrastada eficacia de los tratamientos con sustitutivos de nicotina en la reducción del aumento de peso al dejar de fumar (Parsons, Shraim, Inglis, Aveyard y Hajek, 2009), Prapavessis et al., (2007) realizaron un estudio con una muestra de 142 mujeres inactivas para evaluar el efecto añadido del ejercicio físico sobre dicho tratamiento. Entre aquellas que mantuvieron la abstinencia, no se mostraron diferencias en la ganancia de peso entre los grupos de ejercicio físico y control cuando las participantes recibian tratamiento farmacológico con parches de nicotina. En cambio, cuando las participantes no recibian los parches de nicotina, aquellas que formaban parte del grupo de ejercicio aumentaron significativamente menos de peso que aquellas que recibían únicamente tratamiento cognitivo-conductual $(2,0 \mathrm{~kg}$ de diferencia entre ambos grupos aproximadamente).

Aunque la gran mayoría de los estudios se han focalizado exclusivamente en el efecto del ejercicio aeróbico, un reciente estudio piloto sugiere que el ejercicio de fuerza muscular favorece la reducción tanto del peso como del porcentaje de grasa corporal en personas en tratamiento para dejar de fumar (Ciccolo et al., 2011).

\section{Efecto del ejercicio físico como intervención coadyuvante en el mantenimiento de la abstinencia de tabaco.}

Uno de los estudios que mayor influencia ha tenido en el desarrollo de posteriores investigaciones sobre el efecto del ejercicio físico como co-intervención en el tratamiento de la dependencia de nicotina, tanto por los resultados obtenidos como por su rigor metodológico, fue el desarrollado por Marcus et al., (1999). El objetivo de este ensayo clínico aleatorizado fue evaluar si realizar un programa de ejercicio aeróbico supervisado (45-60 minutos a intensidad vigorosa tres veces por semana durante 12 semanas) junto con el tratamiento cognitivo-conductual habitual para dejar de fumar facilitaba la abstinencia a corto y largo plazo (el grupo control recibía sesiones de contacto junto al tratamiento cognitivo-conductual habitual para dejar de fumar). Realizado en un grupo de mujeres sedentarias (134 en el grupo de ejercicio y 147 en el grupo control), los resultados de este estudio mostraron que el grupo de ejercicio físico presentaba un mayor porcentaje de abstinencia sostenida al finalizar el tratamiento, a los 3 y a los 12 meses de seguimiento. Concretamente, el 11,9\% de las mujeres del grupo de ejercicio en comparación con el 5,4\% de las del grupo control mantenían la abstinencia de forma continuada a los 12 meses de seguimiento. Asimismo, las participantes asignadas al grupo de ejercicio lograron un significativamente mayor número de días sin consumir que las participantes del grupo control tanto al final del tratamiento (30,1 vs. 22,4 dias) como a los 3 meses (65,9 vs. 36,0 dias) y a los 12 meses (127,1 vs. 69,6 días) de seguimiento. Ajustando los resultados por la adherencia al programa, un análisis de regresión logística reveló que las participantes asignadas al grupo de ejercicio presentaban un 35\% menos de probabilidades de recaer en el consumo de tabaco al finalizar el tratamiento, así como un 34\% menos y un 36\% menos a los 3 y 12 meses de seguimiento, respectivamente.

En estudios pilotos previos, este mismo grupo (Marcus, Albrecht, Niaura, Abrams y Thompson, 1991; Marcus et al., 1995) ya había sugerido que el ejercicio físico combinado con el tratamiento cognitivo-conductual para dejar de fumar mejoraba las tasas de abandono y podía ser una estrategia eficaz para facilitar el mantenimiento de la abstinencia. No obstante, ambos estudios se realizaron con muestras pequeñas, hecho que limitaba la generalización de los resultados.

Aunque los resultados de estos tres primeros estudios son globalmente positivos, la aplicabilidad de dicho programa de ejercicio a una población fumadora sedentaria podría verse comprometida (es decir, ser de difícil implementación más allá del ámbito de la investigación), debido a la intensidad del programa aplicado (intensidad vigorosa). El ejercicio aeróbico de intensidad vigorosa, aunque se ha relacionado con una reducción del estrés, por ejemplo a través de la reducción del cortisol en fumadores temporalmente abstinentes (Scerbo, Faulkner, Taylor y Thomas, 2010), también se ha relacionado con un empeoramiento del estado de ánimo (Everson, Daley y Ussher, 2008) y un aumento de los niveles de ansiedad y tensión (Russell, Epstein, Johnston, Block y Blair, 1988) en personas que dejaban de fumar. Además, realizar ejercicio aeróbico a dicha intensidad requiere de un nivel de condición física específica, raramente presente en fumadores sedentarios de larga evolución, que suelen presentar concomitantemente enfermedades crónicas y limitaciones funcionales asociadas al tabaquismo (deRuiter y Faulkner, 2006).

Los estudios más recientes han focalizado la atención en el uso del ejercicio aeróbico de intensidad moderada, en lugar del de intensidad vigorosa, por las ventajas que presenta. Según Marcus et al., (2005), entre estas destacan las siguientes: la mejora de la adherencia, el requerimiento de menor supervisión médica, el menor riesgo de lesión y la posibilidad de realizarse mediante el ejercicio físico más habitual y de más fácil implementación, es decir, caminar.

De esta forma, Marcus et al., (2005) evaluaron en una muestra de 217 mujeres sedentarias la eficacia del ejercicio físico de intensidad moderada, junto al tratamiento cognitivoconductual para dejar de fumar, en el mantenimiento de la abstinencia. La intervención de ejercicio combinaba un programa supervisado más un programa para casa que recomendaba realizar ejercicio de intensidad moderada 5 días a la semana durante un total de 165 minutos semanales. Los resultados de esta intervención no mostraron diferencias entre ambos grupos en la abstinencia sostenida al final del tratamiento, ni en el seguimiento a 3 y 12 meses. No obstante, teniendo en cuenta que no todas las participantes cumplieron las recomendaciones de ejercicio a realizar en casa, un análisis de regresión mostró que aquellas participantes que realizaron más semanas de ejercicio presentaban una mayor probabilidad de estar abstinente.

Más recientemente, un estudio con una muestra total de 60 mujeres sedentarias (Williams et al., 2010) ha evaluado el efecto de un programa de ejercicio aeróbico supervisado de intensidad moderada en combinación con el tratamiento habitual para dejar de fumar. En comparación con el grupo control que realizaba sesiones de contacto, las participantes asignadas al grupo de ejercicio físico (150 minutos de ejercicio aeróbico semanales) tendian a presentar tasas superiores de abstinencia continua tanto al final del tratamiento (8 semanas) como al mes de seguimiento (34,5\% vs. $20 \%$ y $17,2 \%$ vs. $13,3 \%$, respectivamente). 
Tabla 1. Ensayos clínicos aleatorizados que han aplicado un programa de ejercicio físico como intervención coadyuvante en el tratamiento de la dependencia de tabaco

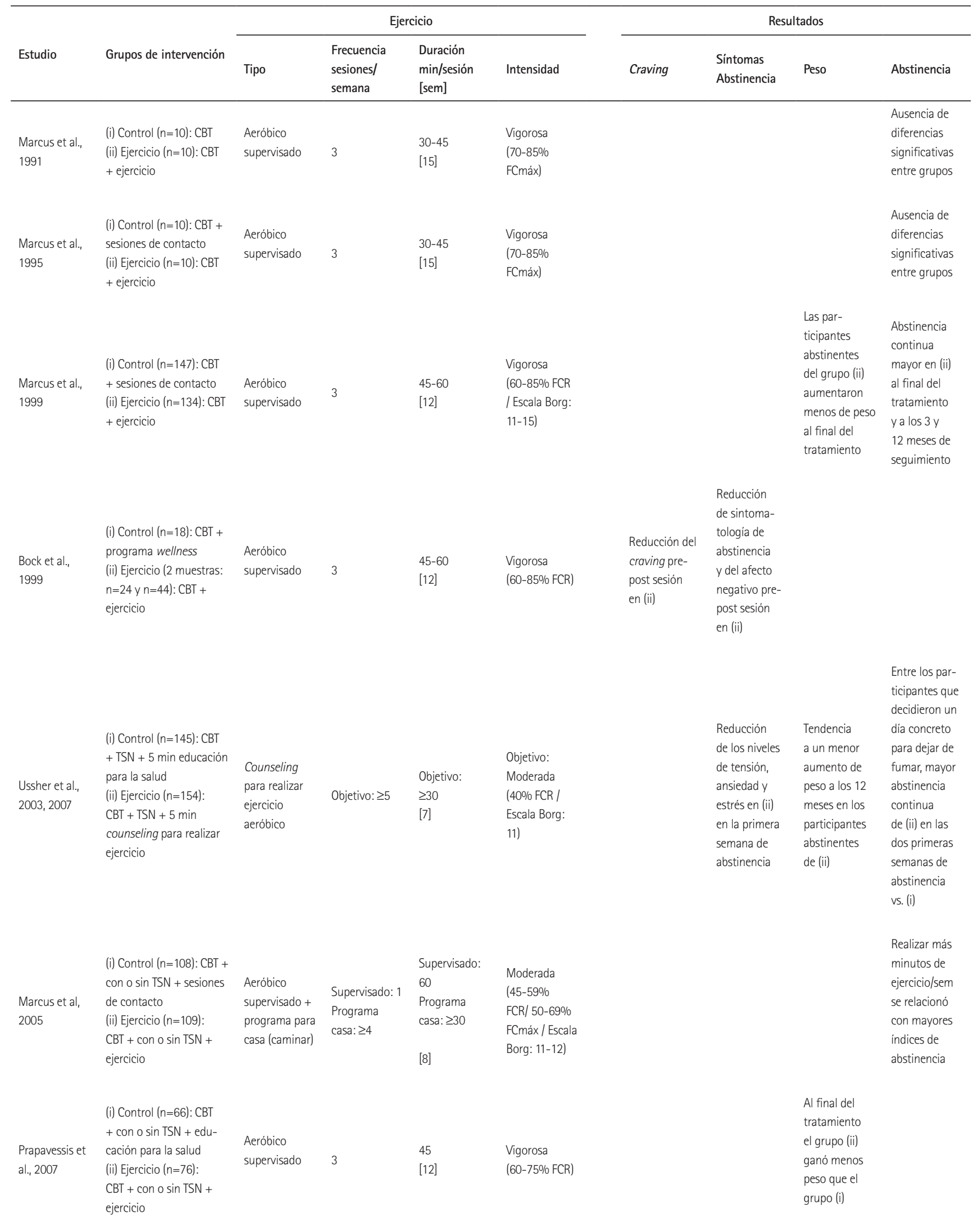




\begin{tabular}{|c|c|c|c|c|c|c|c|c|c|}
\hline \multirow[b]{2}{*}{ Estudio } & \multirow[b]{2}{*}{ Grupos de intervención } & \multicolumn{4}{|c|}{ Ejercicio } & \multicolumn{4}{|c|}{ Resultados } \\
\hline & & Tipo & $\begin{array}{l}\text { Frecuencia } \\
\text { sesiones/ } \\
\text { semana }\end{array}$ & $\begin{array}{l}\text { Duración } \\
\text { min/sesión } \\
\text { [sem] }\end{array}$ & Intensidad & Craving & $\begin{array}{l}\text { Sintomas } \\
\text { Abstinencia }\end{array}$ & Peso & Abstinencia \\
\hline $\begin{array}{l}\text { Williams et al., } \\
\text { 2010, } 2011\end{array}$ & $\begin{array}{l}\text { (i) Control }(n=30) \text { : coun- } \\
\text { seling + TSN + programa } \\
\text { de wellness } \\
\text { (ii) Ejercicio }(n=30) \text { : } \\
\text { counseling + TSN + } \\
\text { ejercicio }\end{array}$ & $\begin{array}{l}\text { Aeróbico } \\
\text { supervisado }\end{array}$ & 3 & $\begin{array}{l}50 \\
{[8]}\end{array}$ & $\begin{array}{l}\text { Moderada } \\
\text { (70\% FCmáx) }\end{array}$ & $\begin{array}{l}\text { Reducción del } \\
\text { craving en el } \\
\text { grupo (ii) pre- } \\
\text { post sesión }\end{array}$ & & & $\begin{array}{l}\text { Tendencia } \\
\text { a mayor } \\
\text { abstinencia } \\
\text { continua en } \\
\text { (ii) al final del } \\
\text { tratamiento } \\
\text { y en el } \\
\text { seguimiento a } \\
1 \text { mes. } \\
\text { Relación } \\
\text { positiva en } \\
\text { el grupo } \\
\text { (ii) entre el } \\
\text { cumplimiento } \\
\text { a las sesiones } \\
\text { de ejercicio y } \\
\text { la abstinencia }\end{array}$ \\
\hline $\begin{array}{l}\text { Bize et al., } \\
2010\end{array}$ & $\begin{array}{l}\text { (i) Control }(n=252) \text { : } \\
\text { counseling + TSN + pro- } \\
\text { grama de salud } \\
\text { (ii) Ejercicio }(n=229) \text { : } \\
\text { counseling + TSN + } \\
\text { ejercicio }\end{array}$ & $\begin{array}{l}\text { Aeróbico } \\
\text { supervisado + } \\
\text { intervención } \\
\text { motivacional } \\
\text { para incre- } \\
\text { mentar el nivel } \\
\text { de actividad } \\
\text { física }\end{array}$ & $\begin{array}{l}\text { Supervisado: } 1 \\
\text { Programa } \\
\text { casa: } \geq 4\end{array}$ & $\begin{array}{l}\text { Supervisado: } \\
60 \\
\text { Programa } \\
\text { casa: } \geq 30 \\
{[9]}\end{array}$ & $\begin{array}{l}\text { Moderada } \\
\text { (Escala Borg: } \\
\text { 11-13) }\end{array}$ & & $\begin{array}{l}\text { Tendencia a } \\
\text { menor sin- } \\
\text { tomatologia } \\
\text { abstinencial } \\
\text { en (ii) }\end{array}$ & $\begin{array}{l}\text { Tendencia no } \\
\text { significativa a } \\
\text { una ganancia } \\
\text { menor de peso } \\
\text { de (ii) vs. (i) a } \\
\text { los } 12 \text { meses } \\
\text { de seguimien- } \\
\text { to en los } \\
\text { abstinentes }\end{array}$ & \\
\hline $\begin{array}{l}\text { Ciccolo et al., } \\
2011\end{array}$ & $\begin{array}{l}\text { (i) Control }(n=13) \text { : } \\
\text { counseling + sesiones de } \\
\text { contacto } \\
\text { (ii) Ejercicio ( } n=13) \text { : } \\
\text { counseling + ejercicio }\end{array}$ & $\begin{array}{l}\text { Fuerza } \\
\text { supervisado }\end{array}$ & 2 & $\begin{array}{l}60 \\
{[12]}\end{array}$ & $\begin{array}{l}65 \%-75 \% \\
\text { fuerza màxima } \\
\text { estimada }\end{array}$ & & & $\begin{array}{l}\text { Reducción del } \\
\text { peso y el \% de } \\
\text { grasa corporal } \\
\text { del grupo (ii) } \\
\text { a los } 3 \text { y } 6 \\
\text { meses }\end{array}$ & $\begin{array}{l}\text { Mayor absti- } \\
\text { nencia pro- } \\
\text { longada en el } \\
\text { grupo (ii) a los } \\
3 \text { y } 6 \text { meses de } \\
\text { seguimiento }\end{array}$ \\
\hline
\end{tabular}

CBT: Terapia Cognitivo Conductual; Escala de Borg: evalúa de forma subjetiva el esfuerzo percibido en una determinada actividad; FCmáx: Frecuencia Cardiaca máxima; FCR: Frecuencia Cardiaca de Reserva; TSN: Terapia Sustitutiva con Nicotina

Otros estudios han mostrado que la intervención combinada mediante terapia sustitutiva de nicotina y un programa de ejercicio físico supervisado facilita la abstinencia de tabaco en mujeres fumadoras sedentarias (Prapavessis et al., 2007) y que un incremento del nivel de actividad física podría ayudar a mantener la abstinencia durante las primeras semanas (Ussher et al., 2003). Asimismo, tal y como muestra un estudio reciente (Ciccolo et al., 2011), el ejercicio de fuerza muscular, al igual que el ejercicio aeróbico, podría mejorar las tasas de abstinencia prolongada, aunque este tipo de ejercicio ha sido poco estudiado hasta la actualidad en el ámbito de los trastornos adictivos.

Mientras que las intervenciones para dejar de fumar que añaden un programa de ejercicio se han desarrollado en personas sedentarias, un estudio realizado en personas fumadoras que ya participaban regularmente en programas de ejercicio físico, sugiere que el ejercicio regular es un factor protector de la recaída en el tratamiento del tabaquismo (Abrantes et al., 2009). En este ensayo clínico, doble ciego, controlado con placebo y de 12 semanas de duración, se compararon 4 intervenciones para dejar de fumar: (a) tratamiento habitual más bupropion, (b) tratamiento habitual más placebo, (c) tratamiento habitual más terapia cognitivo-conductual para la depresión más bupropion $y_{1}(d)$ tratamiento habitual más terapia cognitivo-conductual para la depresión más placebo. Aunque solo el 28\% de la muestra refería en la evaluación basal ser físicamente activa, aquellos que realizaban ejercicio eran más frecuentemente mujeres, presentaban un menor índice de masa corporal, fumaban menos cigarrillos al día, referian presentar una menor urgencia de fumar y un menor número de síntomas depresivos al inicio del tratamiento. Al final del tratamiento e independientemente de la intervención asignada, aquellos que realizaban ejercicio regular presentaban mayores tasas de abstinencia que los sedentarios (40,1\% vs. 32,9\%), aunque estas diferencias no se mantuvieron en el seguimiento a 12 meses. Asimismo, aun siendo similares en función de la práctica o no de ejercicio físico las tasas de abstinencia en los participantes tratados con bupropion, en los que recibian placebo las tasas de abstinencia fueron significativamente superiores en los que practicaban ejercicio físico $(36,9 \%$ vs. $24,1 \%$ ). 
El ejercicio físico en el tratamiento de la dependencia de alcohol y de sustancias psicoactivas ilegales

En la dependencia de alcohol y de las sustancias ilegales, se dispone de un menor número de estudios publicados que evalúen el efecto del ejercicio físico como intervención coadyuvante en el tratamiento de dichos trastornos adictivos. Sin embargo, en los últimos años parece que ha aumentado la investigación en este ámbito, tal como se desprende de la realización de algunos estudios piloto (Brown et al., 2010; Roessler, 2010) o del desarrollo de un importante ensayo clínico (Trivedi et al., 2011).

La tabla 2 muestra un resumen de los estudios que han introducido el ejercicio físico como intervención en el tratamiento del alcohol y/o drogas ilegales y han evaluado su efectividad en la reducción del craving, de la sintomatología de abstinencia o de los niveles de depresión y ansiedad, así como su potencial relevancia como variable facilitadora del mantenimiento de la abstinencia. Una revisión sobre el desarrollo de este ámbito de investigación hasta mediados de la última década puede hallarse en Donaghy y Ussher (2005).

\section{Eficacia del ejercicio físico en el tratamiento de la dependencia de alcohol.}

En el caso de la dependencia del alcohol, Sinyor, Brown, Rostant y Saraganian (1982) estudiaron el efecto de un programa de ejercicio diario durante seis semanas, junto al tratamiento terapéutico habitual en el consumo de dicha sustancia psicoactiva. En este estudio, 58 hombres y mujeres realizaron un programa de ejercicio con aumento progresivo de la intensidad, empezando con aproximadamente 20 minutos de estiramientos y ejercicios de calentamiento, seguido de ejercicios calisténicos y finalmente 12 minutos de trabajo aeróbico. El grupo que realizaba el programa de ejercicio, en comparación con el grupo que no realizaba ejercicio presentó un mayor porcentaje de personas abstinentes de alcohol tanto al final de la intervención como en el seguimiento a los 3 y 18 meses.

Con el objetivo de estudiar si el ejercicio reducía los niveles de ansiedad y depresión en pacientes dependientes de alcohol en tratamiento hospitalario durante 28 dias, Palmer, Vacc y Epstein (1988) seleccionaron consecutivamente dos grupos de pacientes, uno de los cuales realizaba un programa de ejercicio añadido al tratamiento habitual. El programa se realizaba tres veces por semana a intensidad de entre $60-80 \%$ de la frecuencia cardíaca máxima. Cada sesión empezaba con 10 minutos de calentamiento, 20-30 minutos de ejercicio aeróbico y 10 minutos de vuelta a la calma. Aquellos pacientes que participaron en el programa de ejercicio junto al tratamiento habitual para el alcoholismo redujeron sus niveles de ansiedad y depresión en mayor medida que el grupo control.

Recientemente, Brown et al., (2009), superando los problemas metodológicos de los anteriores estudios, desarrollaron una intervención basada en ejercicio aeróbico como complemento al tratamiento ambulatorio para pacientes con dependencia del alcohol. Dicho estudio muestra tanto un incremento del porcentaje de días de abstinencia del consumo de alcohol como una reducción del número de consumiciones al día, al comparar el estado al finalizar el tratamiento y a los 3 meses de seguimiento con la situación basal.

Asimismo, con el objetivo de evaluar la efectividad del ejercicio físico en la reducción de la urgencia para beber alcohol, Ussher, Sampuran, Doshi, West y Drummond (2004) seleccionaron una muestra de hombres y mujeres que habian finalizado un proceso de desintoxicación de alcohol de 10-14 días de duración en régimen hospitalario. Los pacientes fueron asignados a los grupos experimental (10 minutos de ejercicio aeróbico de intensidad moderada) o control (10 minutos de ejercicio aeróbico de intensidad leve). Diez minutos de ejercicio aeróbico de intensidad moderada, en comparación con la misma duración de ejercicio de intensidad baja, produjo una superior reducción del craving de alcohol, tal como ya habian mostrado anteriores estudios realizados en el ámbito de la dependencia de nicotina.

\section{Efecto del ejericicio físico en el tratamiento de la dependencia de sustancias psicoactivas ilegales.}

Hasta la actualidad, existe un número limitado de estudios que analicen el efecto del ejercicio físico como intervención coadyuvante en el tratamiento de la dependencia de sustancias ilegales y/o en policonsumidores (ver tabla 2). No obstante, a continuación expondremos los resultados de algunos estudios recientes que parecen aportar resultados prometedores sobre el efecto beneficioso del ejercicio físico en el tratamiento de la dependencia de dichas sustancias psicoactivas.

Brown et al., (2010) evaluaron la efectividad de un programa de ejercicio aeróbico de intensidad moderada de doce semanas de duración en una muestra de 16 hombres y mujeres consumidores de dos o más sustancias psicoactivas, en tratamiento de su adicción. Al final de la intervención se produjo un incremento del porcentaje de días de abstinencia de alcohol y de otras drogas, respecto a la valoración basal, observándose asimismo una tendencia a la significación estadística a los 3 meses de seguimiento. Además, los participantes que asistieron al 75\% o más de las sesiones presentaron una menor tasa de recaída en el consumo.

En otro estudio piloto (Roessler, 2010), 38 personas con dependencia de sustancias psicoactivas en tratamiento ambulatorio (13\% heroína, 21\% metadona, 23\% otros opiáceos, 23\% analgésicos opiáceos, 10\% cocaína, 13\% anfetaminas y $58 \%$ cannabis) participaron, además del tratamiento habitual, en un programa de ejercicio consistente en sesiones de ejercicio aeróbico y sesiones de deportes de oposición/colaboración (bádminton y voleibol). Los 20 participantes que finalizaron el estudio mejoraron significativamente su nivel de forma física, redujeron su consumo de alcohol y otras drogas y refirieron una disminución tanto de los niveles de urgencia de consumir alcohol y otras drogas como de la sintomatología de abstinencia.

En España, el número de estudios publicados que analicen la efectividad de programas de ejercicio físico en dependientes de sustancias psicoactivas es muy escaso. Dichos estudios se han centrado en pacientes ingresados en comunidades terapéuticas (Pimentel González, 2001) y en internos en centros penitencia- 
rios en tratamiento con metadona (Pérez, 2002). Además, está en marcha un estudio con pacientes en tratamiento con sustitutivos opioides procedentes tanto del ámbito extrapenitenciario como de prisión (March Cerdá, Romero Vallecillo, Danet Danet, Prieto Rodríguez y Martínez Amat, 2011). El interés de estos trabajos se centra en el efecto del ejercicio físico sobre la mejora de la condición física, la reducción o la estabilización de las dosis de las medicaciones administradas (entre ellas los sustitutivos opioides), la mejora de las relaciones interpersonales y, en definitiva, la optimización de la calidad de vida de los participantes.

Tabla 2. Estudios que han aplicado un programa de ejercicio físico como intervención coadyuvante en el tratamiento de la dependencia de alcohol y/o sustancias ilegales

\begin{tabular}{|c|c|c|c|c|c|c|c|c|c|}
\hline \multirow[b]{2}{*}{ Estudio } & \multirow[b]{2}{*}{ Sustancia } & \multirow[b]{2}{*}{ Grupos de intervención } & \multicolumn{4}{|c|}{ Ejercicio } & \multicolumn{3}{|c|}{ Resultados } \\
\hline & & & Tipo & $\begin{array}{l}\text { Frecuencia } \\
\text { sesiones/ } \\
\text { semana }\end{array}$ & $\begin{array}{l}\text { Duración } \\
\mathrm{min} / \mathrm{sesión} \\
\text { [sem] }\end{array}$ & Intensidad & Craving & $\begin{array}{l}\text { Síntomas } \\
\text { Abstinencia }\end{array}$ & Abstinencia \\
\hline $\begin{array}{l}\text { Sinyor et al., } \\
1982\end{array}$ & Alcohol & $\begin{array}{l}\text { (i) Control ( } 2 \text { muestras; } \\
\mathrm{n}=9 \text { y } n=12 \text { ): intervención } \\
\text { para el alcoholismo } \\
\text { (ii) Ejercicio ( } \mathrm{n}=58) \text { : } \\
\text { intervención para el } \\
\text { alcoholismo + ejercicio }\end{array}$ & $\begin{array}{l}\text { Estiramientos } \\
+ \text { Calisténicos } \\
+ \text { Aeróbico } \\
\text { supervisado }\end{array}$ & 7 & $\begin{array}{l}60 \\
{[6]}\end{array}$ & Progresiva & & & $\begin{array}{l}\text { Mayor } \\
\text { abstinencia de } \\
\text { (ii) al final del } \\
\text { tratamiento } \\
\text { y a los } 3 \text { y } \\
18 \text { meses de } \\
\text { seguimiento }\end{array}$ \\
\hline $\begin{array}{l}\text { Palmer et al., } \\
1988\end{array}$ & Alcohol & $\begin{array}{l}\text { (i) Control }(n=26) \text { : } \\
\text { intervención para el } \\
\text { alcoholismo } \\
\text { (ii) Ejercicio }(n=27) \text { : } \\
\text { intervención para el } \\
\text { alcoholismo + ejercicio }\end{array}$ & $\begin{array}{l}\text { Aeróbico } \\
\text { supervisado }\end{array}$ & 3 & $\begin{array}{l}40-50 \\
{[4]}\end{array}$ & $\begin{array}{l}\text { Moderada } \\
\text { (60-80\% } \\
\text { FCmáx) }\end{array}$ & & $\begin{array}{l}\text { Menores } \\
\text { niveles de } \\
\text { ansiedad y } \\
\text { depresión } \\
\text { del grupo (ii) } \\
\text { al final de la } \\
\text { intervención }\end{array}$ & \\
\hline $\begin{array}{l}\text { Brown et al., } \\
2009\end{array}$ & Alcohol & $\begin{array}{l}\text { Ejercicio }(n=19) \text { : } \\
\text { intervención para el } \\
\text { alcoholismo + ejercicio }\end{array}$ & $\begin{array}{l}\text { Aeróbico } \\
\text { supervisado }\end{array}$ & 1 & $\begin{array}{l}35-60 \\
{[12]}\end{array}$ & $\begin{array}{l}\text { Moderada } \\
\text { (55-69\% } \\
\text { FCmáx) }\end{array}$ & & & $\begin{array}{l}\text { Aumento } \\
\text { del número } \\
\text { de dias } \\
\text { abstinentes } \\
\text { al final de la } \\
\text { intervención } \\
\text { y a los } 3 \\
\text { meses de } \\
\text { seguimiento. } \\
\text { Disminución } \\
\text { del número de } \\
\text { consumiciones } \\
\text { diarias a los } \\
3 \text { meses de } \\
\text { seguimiento }\end{array}$ \\
\hline $\begin{array}{l}\text { Brown et al., } \\
2010\end{array}$ & Policonsumo & $\begin{array}{l}\text { Ejercicio }(n=16) \text { : } \\
\text { tratamiento habitual para } \\
\text { dependencia de sustancias } \\
+ \text { ejercicio }\end{array}$ & $\begin{array}{l}\text { Aeróbico } \\
\text { supervisado }\end{array}$ & 1 & $\begin{array}{l}35-60 \\
{[12]}\end{array}$ & $\begin{array}{l}\text { Moderada } \\
\text { (55-69\% } \\
\text { FCmáx) }\end{array}$ & & & $\begin{array}{l}\text { Aumento } \\
\text { del número } \\
\text { de días de } \\
\text { abstinencia } \\
\text { al final de la } \\
\text { intervención } \\
\text { y a los } 3 \\
\text { meses de } \\
\text { seguimiento }\end{array}$ \\
\hline Roessler, 2010 & Policonsumo & $\begin{array}{l}\text { Ejercicio }(n=20) \text { : } \\
\text { tratamiento habitual para } \\
\text { dependencia de sustancias } \\
+ \text { ejercicio }\end{array}$ & $\begin{array}{l}\text { Aeróbico } \\
+ \text { deporte } \\
\text { oposición/ } \\
\text { colaboración }\end{array}$ & 3 & [8-24] & & $\begin{array}{l}\text { Reducción de } \\
\text { la urgencia } \\
\text { por consumir }\end{array}$ & & \\
\hline
\end{tabular}

FCmáx: Frecuencia Cardiaca máxima 


\section{Interés y preferencias hacia el ejercicio físico en pacientes en tratamiento por dependencia de sustancias psicoactivas}

Aunque tuviera interés implementar programas de ejercicio físico como herramienta complementaria en el tratamiento habitual de los trastornos adictivos, en última instancia y tal como sucede con cualquier otra estrategia terapéutica, es importante tener en cuenta la aceptación de la intervención por parte de la población a la que va dirigida. Es por ello que, conocer las preferencias, intereses y barreras percibidas por los potenciales beneficiarios nos puede ayudar a favorecer la participación y la adherencia, adecuando el programa de ejercicio a las necesidades de los diferentes usuarios.

En este sentido, Read et al., (2001) examinaron el interés, el tipo de ejercicio preferido y las barreras percibidas en pacientes con abuso o dependencia de alcohol en tratamiento en un hospital de día. Los pacientes consideraron que realizar ejercicio físico regular tenía, en general, más ventajas que inconvenientes. Entre los beneficios más destacados señalaron el alivio o reducción de la tensión, sentirse menos estresados y tener una actitud más positiva. En cambio, la fatiga al final del día y la baja condición física eran las mayores dificultades o inconvenientes para su realización. Entre dichas barreras para realizar ejercicio regular se hallaban también la baja motivación, el poco tiempo y la percepción de los costes económicos asociados a la práctica de ejercicio. No obstante, más del $75 \%$ de los pacientes se mostró interesado en participar en un programa de ejercicio físico como parte del tratamiento de su dependencia. El tipo de ejercicio más frecuentemente preferido fue caminar, levantar pesas e ir en bicicleta.

En un estudio reciente con pacientes en tratamiento por abuso de sustancias psicoactivas (Abrantes et al., 2011), aunque la mayoría de los pacientes no realizaban ejercicio de forma regular, la gran mayoría se mostraban interesados en participar en un programa de ejercicio específico para personas que se encontraban en tratamiento por su adicción. Un gran número mostró interés en realizar ejercicio de intensidad moderada, en sesiones de entre 30 y 60 minutos, tanto de trabajo aeróbico como de fuerza, con gran variedad de ejercicios en cada sesión, en un gimnasio o al aire libre. En el caso de los hombres destacaba asimismo una preferencia por el ejercicio no supervisado. Al igual que en el estudio de Read et al., (2001) el tipo de ejercicio preferido fue caminar, especialmente en el caso de las mujeres. Los pacientes se mostraron igualmente dispuestos a utilizar podómetros como medida de registro de su evolución, instrumento cuya utilización se ha demostrado que favorece el incremento de la motivación y los niveles de actividad física tanto en población general (Bravata et al., 2007) como en fumadores (Prochaska et al., 2008).

En el caso concreto de la dependencia de opioides, Neale, Nettleton y Pickering (2012) realizaron 40 entrevistas a consumidores de heroína que iniciaban tratamiento. La mitad de los entrevistados habian participado de forma reglada en deportes cuando eran niños o adolescentes, pero dichos niveles de participación se redujeron drásticamente cuando el consumo de heroína se hizo habitual. En el momento de las entrevistas los pacientes, que ya habian empezado a reducir el consumo de heroína o se encontraban en una fase de abstinencia temprana, referían estar retomando el interés por la práctica de ejercicio, interés facilitado por el hecho de que en los centros o servicios en los que estaban en tratamiento organizaban actividades deportivas. En una segunda entrevista, realizada 3 meses más tarde, los participantes en el estudio referian una disminución del consumo de heroína y una mayor práctica de ejercicio físico, pudiendo ser considerados más de la mitad como físicamente activos. Dichos pacientes realizaban frecuentemente actividades no estructuradas como caminar, ir en bicicleta, solos o acompañados por amigos. Aunque hombres y mujeres diferian en la tipología del ejercicio practicado, en ambos casos se mostraban igualmente interesados en mantenerse físicamente activos y consideraban las actividades como divertidas y generadoras de bienestar físico y mental. En relación con la salud mental, los participantes referian que sentirse físicamente activos les ayudaba a reducir los sintomas ansiosos y depresivos y les producía un sentimiento de satisfacción. Asimismo, la realización de ejercicio les ayudaba a mantener la mente apartada de las drogas, a llenar el tiempo libre y a reducir los episodios de aburrimiento. La barrera más importante detectada para realizar ejercicio físico fue el propio consumo de drogas cuando este se hallaba en una fase activa. Otras barreras identificadas fueron una debilitada salud física (por ejemplo, bajo peso, hepatitis C). Entre las barreras psicológicas para el inicio y mantenimiento de la práctica física, destacaban la baja motivación y el no sentirse a gusto acudiendo a un gimnasio público.

\section{Discusión}

Una vez revisada la literatura científica que evalúa la efectividad de programas de ejercicio físico como intervención coadyuvante en el tratamiento de la dependencia de sustancias psicoactivas, puede afirmarse que es cuanto menos prometedora, incluso consistente en el caso de la dependencia de nicotina, la evidencia empírica acumulada hasta la actualidad sobre la utilidad del ejercicio físico como herramienta complementaria en el tratamiento habitual de las adicciones.

En el caso de la dependencia de nicotina, el ejercicio puede ayudar a reducir la sintomatología de abstinencia, el craving y la ganancia de peso. Para conseguir estos resultados es importante determinar de forma precisa el tipo, la frecuencia y la intensidad del ejercicio a realizar. De acuerdo con Ayán Pérez (2009), la duración de las sesiones debería ser de al menos 20 minutos, preferiblemente de intensidad moderada y de tipo aeróbico. En aquellos casos en los que no sea posible realizar este tipo de ejercicio (por ejemplo en situaciones donde uno ha de permanecer sentado durante largo tiempo), ha demostrado ser efectivo realizar trabajos de fuerza con ejercicios isométricos. Aunque este tipo de ejercicio parece ser menos potente que el aeróbico, es una técnica percibida como útil para la reducción inmediata del craving y de la sintomatología de abstinencia al dejar de fumar (Al-Chalabi et al., 2008).

Para prevenir y/o reducir el aumento de peso que se produce en muchas ocasiones al dejar de fumar, la duración y la 
intensidad de las sesiones deberían ser mayores $y$, en cualquier caso, teniendo presente que el control del peso mediante ejercicio físico no es inmediato -al requerir tiempo para compensar el gasto energético- debería adecuarse tanto el volumen y la intensidad de las sesiones (Blundell, Stubbs, Hughes, Whybrow y King, 2003; Chaput et al., 2011), como su frecuencia, preferiblemente diaria. En este sentido, aunque el tipo de ejercicio más estudiado ha sido el aeróbico, también el entrenamiento de fuerza podría ayudar a regular el peso y la grasa corporal, aunque son necesarios nuevos estudios que lo corroboren.

En el caso de la dependencia de alcohol y de sustancias psicoactivas ilegales, aunque el número de estudios es menor que en el caso de la dependencia de nicotina, el ejercicio aeróbico también ha mostrado su efectividad en la reducción de los niveles tanto del craving como de la sintomatología de abstinencia. No obstante, son necesarios más estudios para posibilitar la obtención de resultados más concluyentes.

Debido al efecto del ejercicio físico sobre la activación de los mecanismos de neurotransmisión y las áreas de refuerzo (Dishman et al., 2006; Dishman y 0'Connor, 2009) y su consiguiente función, demostrado en estudios preclínicos, como refuerzo alternativo al consumo de sustancias (Cosgrove, Hunter y Carroll, 2002; Smith y Lynch, 2011; Smith, Schmidt, lordanou y Mustroph, 2008; Zlebnik, Anker, Gliddon y Carroll, 2010), el potencial del ejercicio físico como estrategia de intervención en el tratamiento de la dependencia del alcohol y/o de sustancias ilegales es, como mínimo una hipótesis digna de un mayor número de estudios. De hecho, incluso en el caso de consumidores de alcohol (Murphy, Pagano y Marlatt, 1986) y cannabis (Buchowski et al., 2011) que no se hallaban en tratamiento y no se mostraban interesados en abandonar dicho consumo, el ejercicio aeróbico parece mostrarse efectivo en la reducción del consumo.

Ya sea como intervención para la reducción del consumo, para la disminución de la sintomatología de abstinencia producida tras la retirada de la sustancia o como alternativa al consumo de sustancias psicoactivas, la utilidad del ejercicio físico como estrategia coadyuvante de los tratamientos terapéuticos habituales parece demostrado, al menos en el caso de algunos de los trastornos adictivos. Sin embargo, no es una herramienta utilizada en la práctica habitual, incluso a sabiendas de ser una intervención rentable, flexible, accesible y con pocos efectos secundarios, además de conocerse ampliamente sus efectos beneficiosos sobre la salud física y mental.

Como principales limitaciones de nuestro estudio podemos destacar que, dado que solo se incluyeron artículos publicados en castellano, inglés o francés podrían haberse omitido, en caso de existir, aquellos trabajos publicados en otras lenguas. Asimismo, tampoco se incluyeron eventuales estudios en los que la población de estudio fuera infantil, adolescente o de la tercera edad, por lo que nuestros resultados se centran exclusivamente en población adulta.

Como conclusión, parece razonable y potencialmente útil proponer la incorporación de programas de ejercicio físico en el tratamiento habitual de la dependencia de sustancias psicoactivas. Esta incorporación podría realizarse de diferentes maneras: desde el simple asesoramiento de pautas de acti- vidad física a realizar, mediante counseling o intervenciones motivacionales breves, hasta la incorporación de programas supervisados por especialistas vinculados a los centros de tratamiento. Estableciendo una buena supervisión y asesoramiento por parte de profesionales formados adecuadamente, los usuarios podrian obtener beneficios no despreciables, entre ellos una mejoría de su salud y bienestar y, en definitiva, una mejora de su calidad de vida. Para ello resulta necesario realizar nuevas investigaciones que ayuden a determinar tanto el momento óptimo de inicio de dichas intervenciones como las características adecuadas del ejercicio a practicar, para optimizar su efectividad. Finalmente, destacar que la existencia de un elevado interés, buena aceptación y actitud positiva hacia el ejercicio físico en población adicta, convierte dicha intervención en especialmente viable y factible en el colectivo de personas en tratamiento de su trastorno adictivo.

\section{Conflicto de intereses}

Los autores del artículo manifiestan no tener ningún conflicto de intereses.

\section{Referencias}

Abrantes, A. M., Battle, C. L., Strong, D. R., Ing, E., Dubreuil, M. E., Gordon, A. y Brown, R. A. (2011). Exercise preferences of patients in substance abuse treatment. Mental Health and Physical Activity, 4, 79-87. doi:10.1016/j.mhpa.2011.08.002

Abrantes, A. M., Strong, D. R., Lloyd-Richardson, E. E., Niaura, R., Kahler, C. W. y Brown, R. A. (2009). Regular exercise as a protective factor in relapse following smoking cessation treatment. The American Journal on Addictions, 18, 100-101. doi:10.1080/10550490802545182

Al-Chalabi, L., Prasad, N., Steed, L., Stenner, S., Aveyard, P., Beach, J. y Ussher, M. (2008). A pilot randomised controlled trial of the feasibility of using body scan and isometric exercises for reducing urge to smoke in a smoking cessation clinic. BMC Public Health, 8 , 349. doi:10.1186/1471-2458-8-349

American Psychiatric Association. (2002). Manual diagnóstico y estadístico de los trastornos mentales. Texto revisado. [Diagnostic and Statistical Manual of Mental Disorders. Text Revision] (4ª ed.). Barcelona: Masson.

Aveyard, P. y Ussher, M. (2007). Does exercise in adolescence prevent smoking uptake? Addiction, 102, 1025-1026. doi:10.1111/j.13600443.2007.01919.x

Ayán Pérez, C. (2009). Prescripción de ejercicio en el tratamiento de deshabituación del tabaco. Archivos de Bronconeumologia, 45, 556560. doi:10.1016/j.arbres.2008.08.004

Bize, R., Willi, C., Chiolero, A., Stoianov, R., Payot, S., Locatelli, I. y Cornuz, J. (2010). Participation in a population-based physical activity 
programme as an aid for smoking cessation: A randomised trial. Tobacco Control, 19, 488-494. doi:10.1136/tc.2009.030288

Blair, S. N., LaMonte, M. J. y Nichaman, M. Z. (2004). The evolution of physical activity recommendations: How much is enough? The American Journal of Clinical Nutrition, 79, 913S-920S.

Blundell, J. E., Stubbs, R. J., Hughes, D. A., Whybrow, S. y King, N. A. (2003). Cross talk between physical activity and appetite control: Does physical activity stimulate appetite? The Proceedings of the Nutrition Society, 62, 651-661. doi:10.1079/PNS2003286

Bock, B. C., Marcus, B. H., King, T. K., Borrelli, B. y Roberts, M. R. (1999). Exercise effects on withdrawal and mood among women attempting smoking cessation. Addictive Behaviors, 24, 399-410.

Bravata, D. M., Smith-Spangler, C., Sundaram, V., Gienger, A. L., Lin, N., Lewis, R.,... Sirard, J. R. (2007). Using pedometers to increase physical activity and improve health: A systematic review. JAMA, 298, 2296-2304. doi:10.1001/jama.298.19.2296

Brown, R. A., Abrantes, A. M., Read, J. P., Marcus, B. H., Jakicic, J., Strong, D. R.,... Gordon, A. A. (2009). Aerobic exercise for alcohol recovery: Rationale, program description, and preliminary findings. Behavior Modification, 33, 220-249. doi:10.1177/0145445508329112

Brown, R. A., Abrantes, A. M., Read, J. P., Marcus, B. H., Jakicic, J., Strong, D. R.,... Gordon, A. A. (2010). A pilot study of aerobic exercise as an adjunctive treatment for drug dependence. Mental Health and Physical Activity, 3, 27-34. doi:10.1016/j.mhpa.2010.03.001

Buchowski, M. S., Meade, N. N., Charboneau, E., Park, S., Dietrich, M. S., Cowan, R. L. y Martin, P. R. (2011). Aerobic exercise training reduces cannabis craving and use in non-treatment seeking cannabisdependent adults. PloS One, 6, e17465. doi:10.1371/journal. pone. 0017465

Bull, F. C. y the Expert Working Groups. (2010). Physical activity guidelines in the U.K.: Review and recommendations. Loughborough University: School of Sport, Exercise and Health Sciences, Loughborough University.

Caan, B., Coates, A., Schaefer, C., Finkler, L., Sternfeld, B. y Corbett, K. (1996). Women gain weight 1 year after smoking cessation while dietary intake temporarily increases. Journal of the American Dietetic Association, 96, 1150-1155. doi:10.1016/S00028223(96)00296-9

Chaput, J. P., Klingenberg, L., Rosenkilde, M., Gilbert, J. A., Tremblay, A. y Sjodin, A. (2011). Physical activity plays an important role in body weight regulation. Journal of Obesity, 2011, 360257. doi:10.1155/2011/360257

Ciccolo, J. T., Dunsiger, S. I., Williams, D. M., Bartholomew, J. B., Jennings, E. G., Ussher, M. H.,... Marcus, B. H. (2011). Resistance training as an aid to standard smoking cessation treatment: A pilot study. Nicotine \& Tobacco Research, 13, 756-760. doi:10.1093/ntr/ntr068

Clark, M. M., Hurt, R. D., Croghan, I. T., Patten, C. A., Novotny, P., Sloan, J. A.,... Loprinzi, C. L. (2006). The prevalence of weight concerns in a smoking abstinence clinical trial. Addictive Behaviors, 31, 1144-1152. doi:10.1016/j.addbeh.2005.08.011

Cosgrove, K. P., Hunter, R. G. y Carroll, M. E. (2002). Wheel-running attenuates intravenous cocaine self-administration in rats: Sex differences. Pharmacology, Biochemistry, and Behavior, 73, 663-671.
Daley, A. J. (2002). Exercise therapy and mental health in clinical populations: Is exercise therapy a worthwhile intervention? Advances in Psychiatric Treatment, 8, 262-270.

Daniel, J., Cropley, M., Ussher, M. y West, R. (2004). Acute effects of a short bout of moderate versus light intensity exercise versus inactivity on tobacco withdrawal symptoms in sedentary smokers. Psychopharmacology, 174, 320-326. doi:10.1007/s00213-0031762-x

deRuiter, W. y Faulkner, G. (2006). Tobacco harm reduction strategies: The case for physical activity. Nicotine \& Tobacco Research, 8, 157168. doi:10.1080/14622200500494823

Dimeo, F., Bauer, M., Varahram, I., Proest, G. y Halter, U. (2001). Benefits from aerobic exercise in patients with major depression: A pilot study. British Journal of Sports Medicine, 35, 114-117.

Dishman, R. K., Berthoud, H. R., Booth, F. W., Cotman, C. W., Edgerton, V. R., Fleshner, M. R.,... Zigmond, M. J. (2006). Neurobiology of exercise. Obesity, 14, 345-356.

Dishman, R. K. y O'Connor, P. J. (2009). Lessons in exercise neurobiology: The case of endorphins. Mental Health and Physical Activity, 2, 4-9.

Donaghy, M. E. y Ussher, M. H. (2005). Exercise interventions in drug and alcohol rehabilitation. En G. E. Faulkner y A. H. Taylor (Eds.), Exercise health and mental health: Emerging relationships (pp. 48-69). New York: Routledge.

Durstine, J. L., Moore, G. E., Painter, P. L. y Roberts, S. O. (Eds.) (2009). ACSM's exercise management for persons with chronic diseases and disabilities ( $3^{\text {rd }}$ ed.). Champaign, IL: Human Kinetics.

Everson, E. S., Daley, A. J. y Ussher, M. (2008). The effects of moderate and vigorous exercise on desire to smoke, withdrawal symptoms and mood in abstaining young adult smokers. Mental Health and Physical Activity, 1, 26-31.

Faulkner, G. E. y Taylor, A. H. (2005). Exercise health and mental health: Emerging relationships. New York: Routledge.

Faulkner, G. E. y Taylor, A.H. (2009). Promoting physical activity for mental health: A complex intervention? Mental Health and Physical Activity, 2, 1-3.

Filozof, C., Fernandez Pinilla, M. C. y Fernandez-Cruz, A. (2004). Smoking cessation and weight gain. Obesity Reviews, 5, 95-103. doi:10.1111/ j.1467-789X.2004.00131.x

Fox, K. R. (1999). The influence of physical activity on mental well-being. Public Health Nutrition, 2(Supl. 3a), 411-418.

Generalitat de Catalunya. (2009). Manual d'activitat fisica en atenció primària. Barcelona: Direcció General de Salut Pública (Departament de Salut). Recuperado de: http://www.gencat.cat/ics/professionals/ pdf/manual_activitat_fisica.pdf

Generalitat de Catalunya. (2007). Guia de prescripció d'exercici físic per a la salut (PEFS). Barcelona: Direcció General de Salut Pública (Departament de Salut), Secretaria General de l'Esport (Departament de la Vicepresidencia). Recuperado de: http://www20.gencat.cat/ docs/canalsalut/Home\%20Canal\%20Salut/Professionals/Temes_de_ salut/Activitat_fisica/documents/Guiadeprescripcioversioextensa. pdf 
Goodwin, R. D. (2003). Association between physical activity and mental disorders among adults in the United States. Preventive Medicine, 36, 698-703.

Hajek, P., Stead, L. F., West, R., Jarvis, M. y Lancaster, T. (2009). Relapse prevention interventions for smoking cessation. Cochrane Database of Systematic Reviews, (1), CD003999. doi:10.1002/14651858. CD003999.pub3

Haskell, W. L., Lee, I. M., Pate, R. R., Powell, K. E., Blair, S. N., Franklin, B. A.,... Bauman, A. (2007). Physical activity and public health: Updated recommendation for adults from the American College of Sports Medicine and the American Heart Association. Medicine and Science in Sports and Exercise, 39, 1423-1434. doi:10.1249/ mss.0b013e3180616b27

Holley, J., Crone, D., Tyson, P. y Lovell, G. (2011). The effects of physical activity on psychological well-being for those with schizophrenia: A systematic review. The British Journal of Clinical Psychology, 50, 84-105. doi:10.1348/014466510X496220; $10.1348 / 014466510 \times 496220$

Hughes, J. R. (2006). Clinical significance of tobacco withdrawal. Nicotine \& Tobacco Research, 8, 153-156. doi:10.1080/14622200500494856

Hughes, J. R., Keely, J. y Naud, S. (2004). Shape of the relapse curve and long-term abstinence among untreated smokers. Addiction, 99, 29-38.

Iraurgi, I. y Corcuera, N. (2008). Craving: Concepto, medición y terapéutica. Norte de Salud Mental, 32, 9-22.

Kaczynski, A. T., Manske, S. R., Mannell, R. C. y Grewal, K. (2008). Smoking and physical activity: A systematic review. American Journal of Health Behavior, 32, 93-110. doi:10.5555/ajhb.2008.32.1.93

Kawachi, I., Troisi, R. J., Rotnitzky, A. G., Coakley, E. H. y Colditz, G. A. (1996). Can physical activity minimize weight gain in women after smoking cessation? American Journal of Public Health, 86, 9991004.

Klesges, R. C., Winders, S. E., Meyers, A. W., Eck, L. H., Ward, K. D., Hultquist, C. M.,... Shadish, W. R. (1997). How much weight gain occurs following smoking cessation? A comparison of weight gain using both continuous and point prevalence abstinence. Journal of Consulting and Clinical Psychology, 65, 286-291.

Knubben, K., Reischies, F. M., Adli, M., Schlattmann, P., Bauer, M. y Dimeo, F. (2007). A randomised, controlled study on the effects of a short-term endurance training programme in patients with major depression. British Journal of Sports Medicine, 41, 29-33. doi:10.1136/bjsm.2006.030130

Korhonen, T., Kujala, U. M., Rose, R. J. y Kaprio, J. (2009). Physical activity in adolescence as a predictor of alcohol and illicit drug use in early adulthood: A longitudinal population-based twin study. Twin Research and Human Genetics, 12, 261-268. doi:10.1375/ twin.12.3.261

Kujala, U. M., Kaprio, J. y Rose, R. J. (2007). Physical activity in adolescence and smoking in young adulthood: A prospective twin cohort study. Addiction, 102, 1151-1157. doi:10.1111/j.13600443.2007.01858.x

Lawlor, D. A. y Hopker, S. W. (2001). The effectiveness of exercise as an intervention in the management of depression: Systematic review and meta-regression analysis of randomised controlled trials. BMJ, 322, 763-767.

March Cerdá, J. C., Romero Vallecillo, M., Danet Danet, A., Prieto Rodríguez, M. A. y Martinez Amat, A. (2011). METAHEROGYM: Programa de actividad física e inclusión digital para personas en tratamiento con opioides. RevistaeSalud.Com, 7, 25.

Marcus, B. H., Albrecht, A. E., King, T. K., Parisi, A. F., Pinto, B. M., Roberts, M.,... Abrams, D. B. (1999). The efficacy of exercise as an aid for smoking cessation in women: A randomized controlled trial. Archives of Internal Medicine, 159, 1229-1234.

Marcus, B. H., Albrecht, A. E., Niaura, R. S., Abrams, D. B. y Thompson, P. D. (1991). Usefulness of physical exercise for maintaining smoking cessation in women. The American Journal of Cardiology, 68, 406407.

Marcus, B. H., Albrecht, A. E., Niaura, R. S., Taylor, E. R., Simkin, L. R., Feder, S. I.,... Thompson, P. D. (1995). Exercise enhances the maintenance of smoking cessation in women. Addictive Behaviors, 20, 87-92.

Marcus, B. H., Lewis, B. A., Hogan, J., King, T. K., Albrecht, A. E., Bock, B.,... Abrams, D. B. (2005). The efficacy of moderate-intensity exercise as an aid for smoking cessation in women: A randomized controlled trial. Nicotine \& Tobacco Research, 7, 871-880. doi:10.1080/14622200500266056

Marlatt, G. A. (1985). Lifestyle modification. En G. A. Marlatt y J. R. Gordon (Eds.), Relapse prevention (pp. 280-348). New York: The Guildford Press.

Márquez, S. (1995). Beneficios psicológicos de la actividad física. Revista de Psicología General y Aplicada, 48, 185-206.

Mead, G. E., Morley, W., Campbell, P., Greig, C. A., McMurdo, M. y Lawlor, D. A. (2009). Exercise for depression. Cochrane Database of Systematic Reviews, (3), CD004366. doi:10.1002/14651858. CD004366.pub4

Moore, G. E. (2004). The role of exercise prescription in chronic disease. British Journal of Sports Medicine, 38, 6-7.

Murphy, T. J., Pagano, R. R. y Marlatt, G. A. (1986). Lifestyle modification with heavy alcohol drinkers: Effects of aerobic exercise and meditation. Addictive Behaviors, 11, 175-186.

Neale, J., Nettleton, S. y Pickering, L. (2012). Heroin users' views and experiences of physical activity, sport and exercise. The International Journal on Drug Policy, 23, 120-127. doi:10.1016/j. drugpo.2011.06.004

Palmer, J., Vacc, N. y Epstein, J. (1988). Adult inpatient alcoholics: Physical exercise as a treatment intervention. Journal of Studies on Alcohol, 49, 418-421.

Parsons, A. C., Shraim, M., Inglis, J., Aveyard, P. y Hajek, P. (2009). Interventions for preventing weight gain after smoking cessation. Cochrane Database of Systematic Reviews, (1), CD006219. doi:10.1002/14651858.CD006219.pub2

Pate, R. R., Heath, G. W., Dowda, M. y Trost, S. G. (1996). Associations between physical activity and other health behaviors in a representative sample of US adolescents. American Journal of Public Health, 86, 1577-1581. 
Pate, R. R., Pratt, M., Blair, S. N., Haskell, W. L., Macera, C. A., Bouchard, C.,... King, A. C. (1995). Physical activity and public health. A recommendation from the centers for disease control and prevention and the American College of Sports Medicine. JAMA, 273, 402-407.

Pedersen, B. K. y Saltin, B. (2006). Evidence for prescribing exercise as therapy in chronic disease. Scandinavian Journal of Medicine \& Science in Sports, 16 (Supl. 1), 3-63. doi:10.1111/j.16000838.2006.00520.x

Penedo, F. J. y Dahn, J. R. (2005). Exercise and well-being: A review of mental and physical health benefits associated with physical activity. Current Opinion in Psychiatry, 18, 189-193.

Pérez, F. (2002). Programa de adherencia al ejercicio físico, dirigido a usuarios de Programas de Mantenimiento con Metadona (PMM). Revista Española de Sanidad Penitenciaria, 4, 114-117.

Piasecki, T. M. (2006). Relapse to smoking. Clinical Psychology Review, 26, 196-215. doi:10.1016/j.cpr.2005.11.007

Pimentel González, M. (2001). Análisis de los programas de actividad física y su efecto en la condición física saludable de los sujetos drogodependientes ingresados en las comunidades terapéuticas de Galicia. A Coruña: Universidade da Coruña.

Pinto, B. M., Borrelli, B., King, T. K., Bock, B. C., Clark, M. M., Roberts, M. y Marcus, B. H. (1999). Weight control smoking among sedentary women. Addictive Behaviors, 24, 75-86.

Pisinger, C. y Jorgensen, T. (2007). Weight concerns and smoking in a general population: The Inter99 study. Preventive Medicine, 44, 283289. doi:10.1016/j.ypmed.2006.11.014

Pomerleau, C. S., Zucker, A. N. y Stewart, A. J. (2001). Characterizing concerns about post-cessation weight gain: Results from a national survey of women smokers. Nicotine \& Tobacco Research, 3, 51-60. doi:10.1080/14622200020032105

Prapavessis, H., Cameron, L., Baldi, J. C., Robinson, S., Borrie, K., Harper, T. y Grove, J. R. (2007). The effects of exercise and nicotine replacement therapy on smoking rates in women. Addictive Behaviors, 32, 1416-1432. doi:10.1016/j.addbeh.2006.10.005

Prochaska, J. J., Hall, S. M., Humfleet, G., Muñoz, R. F., Reus, V., Gorecki, J. y Hu, D. (2008). Physical activity as a strategy for maintaining tobacco abstinence: A randomized trial. Preventive Medicine, 47, 215-220. doi:10.1016/j.ypmed.2008.05.006

Read, J. P. y Brown, R. A. (2003). The role of physical exercise in alcoholism treatment and recovery. Professional Psychology: Research and Practice, 34, 49-56.

Read, J. P., Brown, R. A., Marcus, B. H., Kahler, C. W., Ramsey, S. E., Dubreuil, M. E.,... Francione, C. (2001). Exercise attitudes and behaviors among persons in treatment for alcohol use disorders. Journal of Substance Abuse Treatment, 21, 199-206.

Roessler, K. K. (2010). Exercise treatment for drug abuse--a Danish pilot study. Scandinavian Journal of Public Health, 38, 664-669. doi:10.1177/1403494810371249

Russell, P. O., Epstein, L. H., Johnston, J. J., Block, D. R. y Blair, E. (1988). The effects of physical activity as maintenance for smoking cessation. Addictive Behaviors, 13, 215-218.
Scerbo, F., Faulkner, G., Taylor, A. y Thomas, S. (2010). Effects of exercise on cravings to smoke: The role of exercise intensity and cortisol. Journal of Sports Sciences, 28, 11-19. doi:10.1080/02640410903390089

Sinyor, D., Brown, T., Rostant, L. y Seraganian, P. (1982). The role of a physical fitness program in the treatment of alcoholism. Journal of Studies on Alcohol, 43, 380-386.

Smith, M. A. y Lynch, W. J. (2011). Exercise as a potential treatment for drug abuse: Evidence from preclinical studies. Frontiers in Psychiatry, 2, 82. doi:10.3389/fpsyt.2011.00082

Smith, M. A., Schmidt, K. T., Iordanou, J. C. y Mustroph, M. L. (2008). Aerobic exercise decreases the positive-reinforcing effects of cocaine. Drug and Alcohol Dependence, 98, 129-135. doi:10.1016/j. drugalcdep.2008.05.006

Stathopoulou, G., Powers, M. B., Berry, A. C., Smits, J. A. J. y Otto, M. W. (2006). Exercise interventions for mental health: A quantitative and qualitative review. Clinical Psychology: Science and Practice, 13, 179-193.

Taylor, A. H. y Faulkner, G. (2008). Inaugural editorial. Mental Health and Physical Activity, 1, 1-8.

Taylor, A. H., Katomeri, M. y Ussher, M. (2005). Acute effects of selfpaced walking on urges to smoke during temporary smoking abstinence. Psychopharmacology, 181, 1-7. doi:10.1007/s00213005-2216-4

Taylor, A. H. y Ussher, M. H. (2005). Effects of exercise on smoking cessation and coping with withdrawal symptoms and nicotine cravings. En G. Faulkner y A. H. Taylor (Eds.), Exercise health and mental health. Emerging relationships (pp. 135-158). New York: Routledge.

Taylor, A. H., Ussher, M. H. y Faulkner, G. (2007). The acute effects of exercise on cigarette cravings, withdrawal symptoms, affect and smoking behaviour: A systematic review. Addiction, 102, 534-543. doi:10.1111/j.1360-0443.2006.01739.x

Taylor, C. B., Sallis, J. F. y Needle, R. (1985). The relation of physical activity and exercise to mental health. Public Health Reports, 100, 195-202.

Travier, N., Agudo, A., May, A. M., Gonzalez, C., Luan, J., Wareham, N. J.,... Peeters, P. H. (2012). Longitudinal changes in weight in relation to smoking cessation in participants of the EPIC-PANACEA study. Preventive Medicine, 54, 183-192. doi:10.1016/j.ypmed.2011.09.003

Trivedi, M. H., Greer, T. L., Grannemann, B. D., Church, T. S., Somoza, E., Blair, S. N.,... Nunes, E. (2011). Stimulant reduction intervention using dosed exercise (STRIDE) - CTN 0037: Study protocol for a randomized controlled trial. Trials, 12, 206. doi:10.1186/1745-621512-206

United States Department of Health and Human Services. (1990). The health benefits of smoking cessation. A report of the surgeon general. Washington D.C.: U.S. Department of Health and Human Services. Recuperado de: http://profiles.nIm.nih.gov/ps/access/ NNBBCV.pdf

United States Department of Health and Human Services. (1996). Physical activity and health: A report of the surgeon general. Atlanta: U.S. Department of Health and Human Services, Centers 
for Disease Control and Prevention, National Center for Chronic Disease Prevention and Health Promotion. Recuperado de: http:// www.cdc.gov/nccdphp/sgr/pdf/sgrfull.pdf

United States Department of Health and Human Services. (2001). Women and smoking. surgeon general's report. Washington D.C.: U.S. Department of Health and Human Services. Recuperado de: http://www.cdc.gov/tobacco/data_statistics/sgr/2001/complete_ report/index.htm

United States Department of Health and Human Services. (2008a). Physical activity guidelines for americans. Washington DC: U.S. Department of Health and Human Services. Recuperado de: http:// www.health.gov/paguidelines/pdf/paguide.pdf

United States Department of Health and Human Services. (2008b). Physical activity guidelines advisory committee report. Washington D.C.: U.S. Department of Health and Human Service. Recuperado de: http://www.health.gov/paguidelines/report/pdf/CommitteeReport. pdf

Ussher, M., Cropley, M., Playle, S., Mohidin, R. y West, R. (2009). Effect of isometric exercise and body scanning on cigarette cravings and withdrawal symptoms. Addiction, 104, 1251-1257. doi:10.1111/ j.1360-0443.2009.02605.x

Ussher, M., Nunziata, P., Cropley, M. y West, R. (2001). Effect of a short bout of exercise on tobacco withdrawal symptoms and desire to smoke. Psychopharmacology, 158, 66-72. doi:10.1007/ s002130100846

Ussher, M., Sampuran, A. K., Doshi, R., West, R. y Drummond, D. C. (2004). Acute effect of a brief bout of exercise on alcohol urges. Addiction, 99, 1542-1547. doi:10.1111/j.1360-0443.2004.00919.x

Ussher, M., Taylor, A. y Faulkner, G. (2012). Exercise interventions for smoking cessation. Cochrane Database of Systematic Reviews, (1), CD002295. doi:10.1002/14651858.CD002295.pub4

Ussher, M., West, R., Doshi, R. y Sampuran, A. K. (2006). Acute effect of isometric exercise on desire to smoke and tobacco withdrawal symptoms. Human Psychopharmacology, 21, 39-46. doi:10.1002/ hup.744

Ussher, M., West, R., McEwen, A., Taylor, A. y Steptoe, A. (2003). Efficacy of exercise counselling as an aid for smoking cessation: A randomized controlled trial. Addiction, 98, 523-532.

Ussher, M., West, R., McEwen, A., Taylor, A. y Steptoe, A. (2007). Randomized controlled trial of physical activity counseling as an aid to smoking cessation: 12 month follow-up. Addictive Behaviors, 32, 3060-3064. doi:10.1016/j.addbeh.2007.04.009

Whitelaw, S., Swift, J., Goodwin, A. y Clark, D. (2008). Physical activity and mental health: The role of physical activity in promoting mental wellbeing and preventing mental health problems: an evidence briefing. Edinburgh: NHS Health Scotland.

Williams, D. M., Dunsiger, S., Whiteley, J. A., Ussher, M. H., Ciccolo, J. T. y Jennings, E. G. (2011). Acute effects of moderate intensity aerobic exercise on affective withdrawal symptoms and cravings among women smokers. Addictive Behaviors, 36, 894-897. doi:10.1016/j. addbeh.2011.04.001

Williams, D. M., Whiteley, J. A., Dunsiger, S., Jennings, E. G., Albrecht, A. E., Ussher, M. H.,... Marcus, B. H. (2010). Moderate intensity exercise as an adjunct to standard smoking cessation treatment for women: A pilot study. Psychology of Addictive Behaviors, 24, 349-354. doi:10.1037/a0018332

Wolff, E., Gaudlitz, K., von Lindenberger, B. L., Plag, J., Heinz, A. y Strohle, A. (2011). Exercise and physical activity in mental disorders. European Archives of Psychiatry and Clinical Neuroscience, 261 (Supl. 2), S186-91. doi:10.1007/s00406-011-0254-y

Wright, K. A., Everson-Hock, E. S. y Taylor, A. H. (2009). The effects of physical activity on physical and mental health among individuals with bipolar disorder: A systematic review. Mental Health and Physical Activity, 2, 86-94.

Zlebnik, N. E., Anker, J. J., Gliddon, L. A. y Carroll, M. E. (2010). Reduction of extinction and reinstatement of cocaine seeking by wheel running in female rats. Psychopharmacology, 209, 113-125. doi:10.1007/s00213-010-1776-0 
